Vietnam Journal of Mechanics, VAST, Vol.39, No. 2 (2017), pp. 177-189

DOI:10.15625/0866-7136/10208

\title{
SEISMIC FRAGILITY CURVES BASED ON THE PROBABILITY DENSITY EVOLUTION METHOD
}

\author{
Cong-Thuat Dang ${ }^{1, *}$, Thien-Phu Le ${ }^{2}$, Pascal Ray ${ }^{3}$ \\ ${ }^{1}$ University of Science and Technology - The University of Danang, Vietnam \\ ${ }^{2}$ Université Clermont Auvergne, Sigma-Clermont, Institut Pascal, France \\ ${ }^{3}$ École Nationale Supérieure des Mines de Saint Etienne, France \\ *E-mail: dangcongthuat@dut.udn.vn \\ Received September 15, 2016
}

\begin{abstract}
A seismic fragility curve that shows the probability of failure of a structure in function of a seismic intensity, for example peak ground acceleration (PGA), is a powerful tool for the evaluation of the seismic vulnerability of the structures in nuclear engineering and civil engineering. The common assumption of existing approaches is that the fragility curve is a cumulative probability log-normal function. In this paper, we propose a new technique for construction of seismic fragility curves by numerical simulation using the Probability Density Evolution Method (PDEM). From the joint probability density function between structural response and random variables of a system and/or excitations, seismic fragility curves can be derived without the log-normal assumption. The validation of the proposed technique is performed on two numerical examples.
\end{abstract}

Keywords: Seismic fragility curve, probability density evolution method, log normal probability law, reliability, failure.

\section{INTRODUCTION}

A seismic fragility curve expresses the probability of failure or damage of a structure or a mechanical system due to earthquakes as a function of a ground motion index; for instance, peak ground acceleration (PGA), peak ground velocity (PGV), spectral acceleration at a period of interest (PS $\mathrm{a})$, and so on.

Let $A$ be a chosen ground motion index. The fragility curve $F_{r}(a)$ is seen as the conditioned probability of failure or a damage state given that $A=a$

$$
F_{r}(a)=P\left[X \geq x_{0} \mid A=a\right],
$$

where the failure or the specific damage state is reached when the structural response $X$ exceeds a critical limit $x_{0}$. Seismic hazard, fragility curves and dominant accident sequences which lead to core damage in a plant are three ingredients for a seismic probabilistic risk assessment (PRA) in nuclear engineering application [1]. Fragility curves are

(C) 2017 Vietnam Academy of Science and Technology 
also applied to different structural types in the civil engineering field e.g. buildings [2], bridges [3, 4], special structures: chimney [5], piping systems [6], tunnels [7], highway and railway embankments and cuts [8], etc. They are useful for the design of a new structure, and for an existing one they are helpful for seismic retrofitting decisions, disaster response planning and quick loss estimation [9].

Fragility curves can be obtained using one of three approaches: (i) engineering judgment, (ii) empirical approach, (iii) numerical simulation. These approaches can also be used conjunctively. This paper focuses on the ways of constructing seismic fragility curves using numerical simulations, where there are three popular methods (i) scaled seismic intensity (SSI) [3,9,10], (ii) maximum likelihood estimation (MLE) [11-13] and (iii) probabilistic seismic demand model/probabilistic seismic capacity model (PSDM/PSCM) $[4,14]$. A comparative study on these methods can be found in reference [15]. It should be noted that most of existing methods are based on the common assumption of log-normal shape of the curves

$$
F_{r}\left(a ; A_{m}, \beta\right)=\Phi\left[\frac{\ln \left(a / A_{m}\right)}{\beta}\right],
$$

where $\Phi($.$) is the standard Gaussian cumulative distribution function while A_{m}$ and $\beta$ are respectively median and logarithmic standard deviation. The log-normal assumption given in Eq. (2) greatly simplifies the original problem defined in Eq. (1). All existing methods lead therefore to identify two parameters: $A_{m}$ and $\beta$.

Recently, the probability density evolution method (PDEM) has been developed by Li and Chen [16-20], which was used for dynamic response analysis of structures involving random parameters and stochastic excitations. In this paper, based on PDEM, we propose a new method for the development of seismic fragility curves. The proposed method allows to derive the fragility curves without log-normal shape assumption. A brief background of the PDEM is first described in Section 2. Section 3 is then devoted to the development of the method for building fragility curves. From analytical formulations, a step-by-step practical procedure of the method is deduced. The validation of the proposed method is followed in Section 4 with two numerical examples. Finally, some conclusions on the obtained results and perspectives of this work are given.

\section{PROBABILITY DENSITY EVOLUTION METHOD}

For the sake of completeness, this section gives a basic presentation of the probability density evolution method together with the most relevant properties to the proposed method for constructing seismic fragility curves. More details of the PDEM can be found in references [16-20].

A mechanical system after a discretization (by the finite element analysis for instance) can be represented by an equivalent system of $n$ degrees of freedom. Its response is governed by the following equation

$$
\mathbf{M}(\mathbf{Z}) \ddot{\mathbf{x}}(t)+\mathbf{C}(\mathbf{Z}) \dot{\mathbf{x}}(t)+\mathbf{K}(\mathbf{Z}) \mathbf{x}(t)=\mathbf{f}(\mathbf{Z}, t),
$$

where $\ddot{\mathbf{x}}, \dot{\mathbf{x}}, \mathbf{x}$ are $n \times 1$ acceleration, velocity and displacement vectors while $\mathbf{M}, \mathbf{C}, \mathbf{K}$ are $n \times n$ mass, damping and stiffness matrix, respectively. $\mathbf{f}$ is $n \times 1$ load vector and $\mathbf{Z}$ is $n_{Z} \times 1$ vector of $n_{Z}$ random parameters that reflect the uncertainty in excitations, in 
geometry and mechanical properties of structures. In the case of earthquake excitation, the load vector in Eq. (3) is substituted by

$$
\mathbf{f}(\mathbf{Z}, t)=-\mathbf{M}(\mathbf{Z}) \mathbf{i} a_{g}(\mathbf{Z}, t),
$$

where $\mathbf{i}$ is the influence vector and $a_{g}(\mathbf{Z}, t)$ is ground acceleration.

Assume that random parameters $\mathbf{Z}$ are represented by a known probability density functions $p_{\mathbf{Z}}(\mathbf{z})$, where $\mathbf{Z}=\left(Z_{1}, Z_{2}, \ldots, Z_{n_{Z}}\right)$. For a deterministic value of $\mathbf{z}$ (a trial of $\mathbf{Z})$, the corresponding responses $x(\mathbf{z}, t), \dot{x}(\mathbf{z}, t), \ddot{x}(\mathbf{z}, t)$ of the mechanical system are easily obtained by a structural dynamic analysis. Li and Chen [16-20] show that the joint probability density function $p_{X \mathbf{Z}}(x, \mathbf{z}, t)$ is the solution of the following partial differential equation

$$
\frac{\partial p_{X \mathbf{Z}}(x, \mathbf{z}, t)}{\partial t}+\dot{x}(\mathbf{z}, t) \frac{\partial p_{X \mathbf{Z}}(x, \mathbf{z}, t)}{\partial x}=0,
$$

where $\dot{x}(\mathbf{z}, t)$ is the velocity obtained by structural dynamic analysis and the initial condition is written as

$$
p_{X \mathbf{Z}}(x, \mathbf{z}, t)=\delta\left(x-x\left(t_{0}\right)\right) p_{\mathbf{Z}}(\mathbf{z}) \text { pour } t=t_{0},
$$

with $\delta(\cdot)$ is the Dirac function. The numerical resolution of Eq. (5) gives the joint probability density function $p_{X \mathbf{Z}}(x, \mathbf{z}, t)$ and from this result, it can deduce the probability density $p_{X}(x, t)$ of the response $X(t)$.

$$
p_{X}(x, t)=\int_{\Omega_{\mathbf{Z}}} p_{X \mathbf{Z}}(x, \mathbf{z}, t) d \mathbf{z}
$$

where $\Omega_{\mathbf{Z}}$ is the domain of distribution of random variables $\mathbf{Z}$.

For the dynamic reliability assessment, it can be treated in associating Eq. (5) with the boundary condition reflecting the failure domain $\Omega_{f}$

$$
p_{X \mathbf{Z}}(x, \mathbf{z}, t)=0 \text { si } x \in \Omega_{f},
$$

$\Omega_{f}$ is in general defined via a limit-state function

$$
G(\mathbf{z}, t)=\left(x_{0}-|x(\mathbf{z}, t)|\right) \leq 0 .
$$

The resolution of Eq. (5) with the initial condition in Eq. (6) and the absorbing boundary condition in Eq. (8) provides the joint probability density of $X$ and $\mathbf{Z}$, denoted as $\breve{p}_{X \mathbf{Z}}(x, \mathbf{z}, t)$. It should note that the joint probability $\breve{p}_{X \mathbf{Z}}(x, \mathbf{z}, t)$ is defined by $X$ and $\mathbf{Z}$, in which $X$ is limited in the safety domain $\Omega_{s}$. So $\breve{p}_{X \mathbf{Z}}(x, \mathbf{z}, t)$ can be called the joint probability density in safety domain and the dynamic reliability can be deduced by

$$
R(t)=\int_{\Omega_{s}} \int_{\Omega_{\mathbf{Z}}} \breve{p}_{X \mathbf{Z}}(x, \mathbf{z}, t) d x d \mathbf{z} .
$$

\section{SEISMIC FRAGILITY CURVE BASED ON PDEM}

\subsection{Analytical development}

To facilitate the writing of formulas, the vector of random parameters defined in section 2 is separated into two parts (i) a random variable characterizing the seismic intensity (the peak ground acceleration (PGA) used in this study), denoted by $A$ and 
(ii) other random variables $Z$. This new notation implies the unknown of the problem in Eq. (5) is $p_{X \mathbf{Z} A}(x, \mathbf{z}, a, t)$.

The joint probability density function conditioned by safety domain $\breve{p}_{X \mathbf{Z} A}(x, \mathbf{z}, a, t)$ is determined by the resolution of the problem in Eq. (5) with the initial condition in Eq. (6) and the absorbing boundary condition in Eq. (8).

The probability density of the seismic intensity $A$ in the safety domain $\Omega_{S}$ is evaluated by the integral

$$
\breve{p}_{A}(a, t)=\int_{\Omega_{S}} \int_{\Omega_{\mathbf{Z}}} \breve{p}_{X \mathbf{Z} A}(x, \mathbf{z}, a, t) d \mathbf{z} d x .
$$

As the failure domain $\Omega_{f}$ and the safety domain $\Omega_{S}$ are disjoint, the probability density in failure domain $\hat{p}_{A}(a, t)$ can be obtained by

$$
\hat{p}_{A}(a, t)=p_{A}(a)-\breve{p}_{A}(a, t),
$$

where $p_{A}(a)$ is the initial probability distribution of random parameter $A$ that is assumed invariable in time. Note that $\hat{p}_{A}(a, t)$ means the joint probability density of the event $X \geq x_{0}$ and the event $a \leq A \leq a+d a$ at time $t$. The joint probability is evaluated by its density

$$
P\left[X \geq x_{0}, a \leq A \leq a+d a, t\right]=\hat{p}_{A}(a, t) d a,
$$

and

$$
P[a \leq A \leq a+d a]=p_{A}(a, t) d a .
$$

According to the definition in Eq. (1), instantaneous fragility curve $F_{r}(a ; t)$ is the conditional probability $P\left[X \geq x_{0} \mid A=a, t\right]$. It can be calculated through the Bayes's relation as

$$
\begin{aligned}
F_{r}(a ; t)=\left[X \geq x_{0} \mid A=a, t\right] & \approx P\left[X \geq x_{0} \mid a \leq A \leq a+d a, t\right] \\
& =\frac{P\left[X \geq x_{0}, a \leq A \leq a+d a, t\right]}{P[a \leq A \leq a+d a]} .
\end{aligned}
$$

In substituting Eq. (13) and Eq. (14) into Eq. (15), instantaneous fragility is thus obtained by

$$
F_{r}(a ; t)=\frac{\hat{p}_{A}(a, t) d a}{p_{A}(a, t) d a}=\frac{\hat{p}_{A}(a, t)}{p_{A}(a, t)}=1-\frac{\breve{p}_{A}(a, t)}{p_{A}(a)} .
$$

From Eq. (16), it is possible to build a fragility surface $F_{r}(a, t)$. Because the fragility curve is considered for the whole earthquake duration, thus we propose to take the maximum value of $F_{r}(a, t)$ in time $t$

$$
F_{r}(a)=\max _{t} F_{r}(a ; t)
$$

\subsection{Practical procedure}

To facilitate the application, the proposed method is presented by a step-by-step practical procedure. It consists in two main steps bellow: 
Step 1. Determine the joint probability density function $\breve{p}_{X Z A}(x, \mathbf{z}, a, t)$

- Discretize the random variables $Z$ and $A$ from their known probability density functions, to obtain representative points $\mathbf{z}$ and $a$.

- Analyze the dynamic response of structures (by finite element method for instance), for each pair of values $\mathbf{z}$ and $a$. The response velocity $\dot{x}(\mathbf{z}, a, t)$ is to retrieve.

- Solve the partial differential equation in Eq. (5) with the initial condition in Eq. (6) and the boundary condition in Eq. (8) to obtain $\breve{p}_{X \mathbf{Z} A}(x, \mathbf{z}, a, t)$. The numerical scheme TVD (Total Variation Diminishing) can be used as suggested by $\mathrm{Li}$ and Chen $[16,18]$.

\section{Step 2. Build the fragility curve $F_{r}(a)$}

- Compute $\breve{p}_{A}(a, t)$ by the integral given in Eq. (11).

- Deduce the fragility surface $F_{r}(a, t)$ according to Eq. (16).

- Obtain the fragility curve $F_{r}(a)$ according to Eq. (17).

\section{APPLICATIONS}

The practical procedure is now applied for two numerical examples: non-linear oscillators and a non-linear steel frame. Time ground accelerations were either synthetic or real data. In the case of oscillators they were generated using the Boore's model [21] while they were real records in the case of frame example.

\subsection{Synthetic ground motion histories}

The stochastic method proposed by Boore [21] assumes that ground motion is distributed with random phase over a time duration related to earthquake size and propagation distance. The ground motion is characterized by its spectrum - this is where the physics of earthquake process and wave propagation are contained, usually encapsulated and put into the form of simple equations. The total spectrum of the motion at a site $S\left(M_{0}, R, f\right)$ is considered as a combination of earthquake source $(E)$, path $(P)$, site $(G)$ and type of motion $(I)$.

$$
S\left(M_{0}, R, f\right)=E\left(M_{0}, f\right) P(R, f) G(f) I(f),
$$

where $M_{0}$ is the seismic moment that is related to the seismic magnitude $M$ by

$$
M=\frac{2}{3} \log M_{0}-10,
$$

$R$ is the distance from source to site and $f$ is frequency. The source $E\left(M_{0}, f\right)$ : is based on the source spectral shape AS00 [22]. The path $P(R, f)$ accounts the effects of geometrical spreading, attenuation and the increase of duration with distance due to wave propagation and scattering. The site $G(f)$ accounts the effects due to local site geology and is separated to amplification $A(f)$ and attenuation $D(f): G(f)=D(f) A(f)$. The motion type $I(f)$ for acceleration is defined as $I(f)=(2 \pi f)^{2}$.

Given the spectrum motion at a site, Boore [21] suggest the simulation of ground motions by 6 steps: (i) white noise (Gaussian or uniform) is generated for a duration 
given by the duration of the motion; (ii) this noise is then windowed; (iii) the windowed noise is transformed into frequency domain; (iv) the spectrum is normalized by the square-root of the mean square amplitude spectrum; (v) the normalized spectrum is multiplied by the ground motion spectrum $S\left(M_{0}, R, f\right)$; (vi) the resulting spectrum is transformed back to time domain to obtain a time history of ground motions.

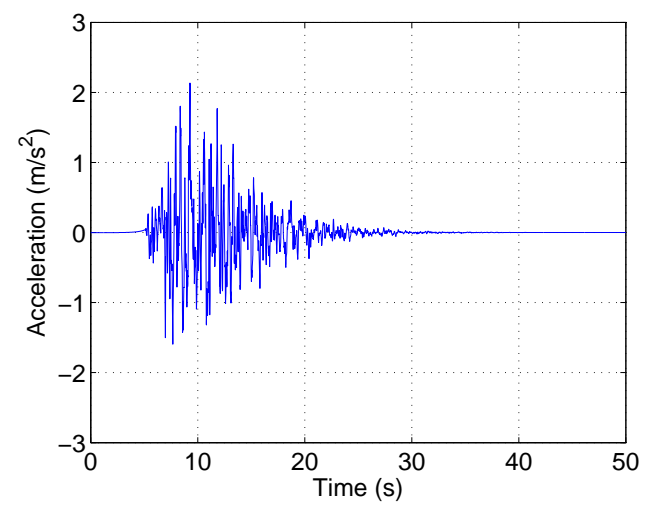

Fig. 1. Simulated ground acceleration with Boore's model $(M=7$ and $R=9 \mathrm{~km})$

In order to generate time acceleration series for the numerical examples, we implemented the stochastic method in Matlab software [23]. Fig. 1 shows an acceleration time series of the model for magnitude $M=7$ and $R=9 \mathrm{~km}$.

\subsection{Non-linear oscillators}

A Bouc-Wen non-linear oscillator used in reference [10] and a Coulomb non-linear oscillator were considered here.

$$
\begin{aligned}
& \text { Bouc-Wen : } \ddot{x}(t)+2 \zeta \omega_{0} \dot{x}(t)+\omega_{0}^{2}(\alpha x(t)+(1-\alpha) w(t))=-a(t), \\
& \text { with } \dot{w}(t)=C_{1} \dot{x}(t)-C_{2}|\dot{x}(t)||w(t)|^{n_{d}-1} w(t)-C_{3} \dot{x}(t)|w(t)|^{n_{d}} . \\
& \text { Coulomb }: \ddot{x}(t)+\mu g \operatorname{Sgn}(\dot{x}(t))+\omega_{0}^{2} x(t)=-a(t),
\end{aligned}
$$

where $\omega_{0}(\mathrm{rad} / \mathrm{s})$ is the natural angular frequency; $\zeta$ is the damping ratio; for BoucWen behavior: $w(t)$ is the hysteretic displacement and $\alpha, C_{1}, C_{2}, C_{3}, n_{d}$ are constants; for Coulomb's model: $\mu$ is the damping fiction coefficient, $a(t)$ is ground acceleration and $g$ is gravitational acceleration. Fig. 2 shows the two models. Numerical parameter values were inspired by Kafali et Grigoriu [10]: $\omega_{0}=5.9 \mathrm{rad} / \mathrm{s}, \zeta=2 \%, C_{1}=1, C_{2}=C_{3}=$ $0.5 \mathrm{~cm}^{n_{d}}, \alpha=0.1, n_{d}=1$ for the Bouc-Wen oscillator; $\mu=0.01$ and $g=9.81 \mathrm{~m} / \mathrm{s}^{2}$ for the Coulomb oscillator.

Oscillator responses under seismic excitations were obtained with the Runge-Kutta algorithm in Matlab software [23]. The failure of an oscillator was verified by comparing displacement $x(t)$ with a displacement limit $x_{0}$ i.e., $X=\max _{t}|x(t)|$. The displacement limit $x_{0}=7 \mathrm{~cm}$ was chosen in this example. 


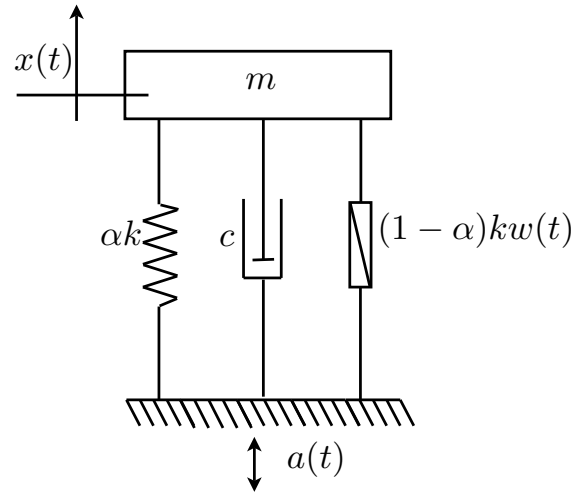

(a)

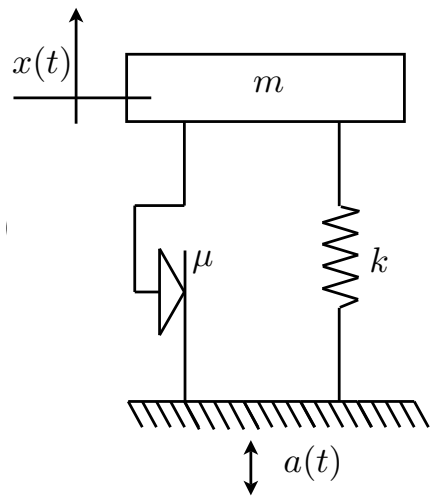

(b)

Fig. 2. Bouc-Wen oscillator (a) and Coulomb oscillator (b)

The probability density function $p_{A}(a)$ is log-normal with mean value equal to $1.4 \mathrm{~m} / \mathrm{s}^{2}$ and $\operatorname{logarithmic}$ standard deviation equal to $0.4 \log \left(\mathrm{m} / \mathrm{s}^{2}\right)$. In order to apply the proposed method, the parameter $A$ was discretized in 30 values and combined with 20 ground acceleration histories. A total of $30 \times 20=600$ simulations was thus used for structural analysis. For comparison purpose, the Monte Carlo Simulation method with $10^{5}$ simulations was also performed.

The reliability $R(t)$ of the oscillators were determined by the PDEM and MCS methods. The obtained results in the time interval $[0,20 \mathrm{sec}]$ are shown in Fig. 3. There is a good agreement between the results of two methods. The validation of PDEM and its numerical implementation were verified.

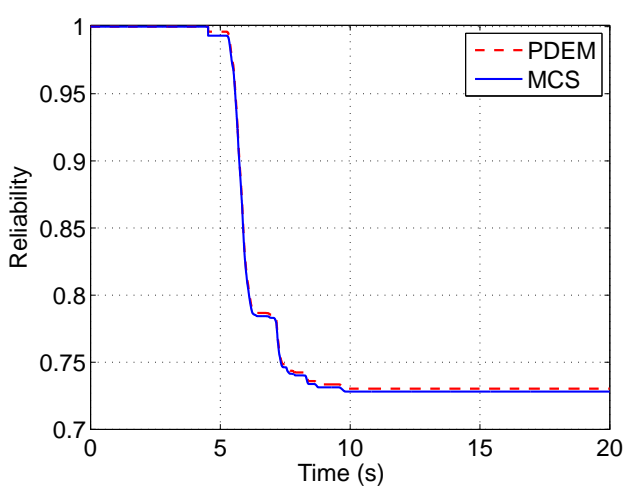

(a)

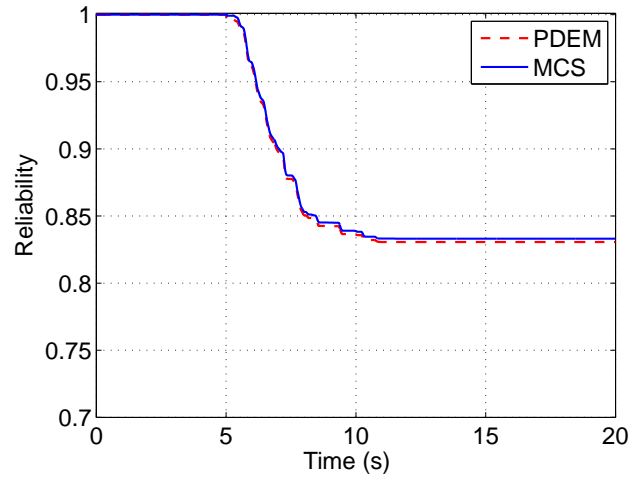

(b)

Fig. 3. Reliability of Bouc-Wen oscillator (a) and Coulomb oscillator (b) 


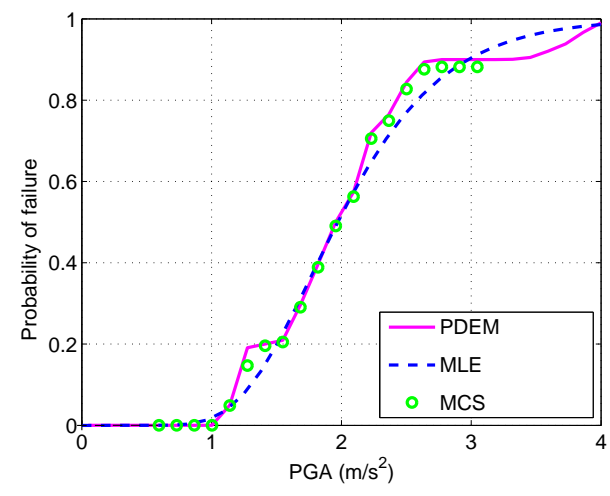

(a)

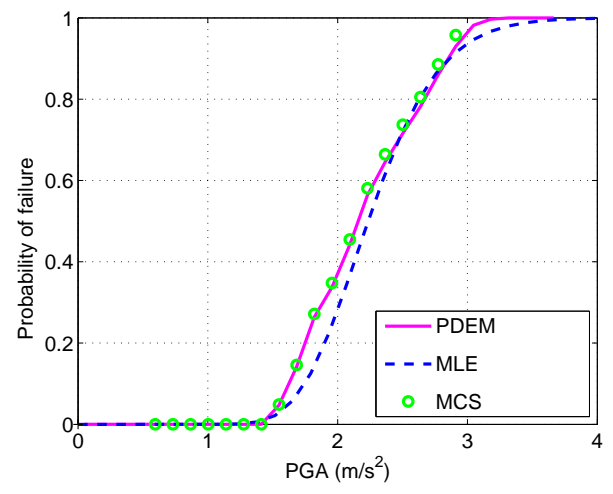

(b)

Fig. 4. Fragility curves of Bouc-Wen oscillator (a) and Coulomb oscillator (b)

Fragility curves obtained by the proposed method and also the existing MLE method are given in Fig. 4. Note that 600 simulations were used for the PDEM and MLE methods while $10^{5}$ simulations for the MCS method in which accelerations were organized in PGA intervals $a_{j}:\left[a_{j}-0.15 ; a_{j}+0.15\right] \mathrm{m} / \mathrm{s}^{2}$. It can be noted that the fragility curves obtained by the proposed method are very close to the MCS results. In comparison with the MLE method, the proposed method is better because superior with the same number of simulations their fragility curves are closer to the MCS results than those derived from the MLE method.

\subsection{Non-linear frame structure}

In this example, a 3-storey 3-span steel frame structure that was used in reference [24], was considered. The frame model is presented in Fig. 5 with the dimension values : storey-height $H=3 \mathrm{~m}$, span-length $L=5 \mathrm{~m}$. The steel material has a non-isotropic non-linear behavior following the Giuffre-Menegotto-Pinto model. The initial elastic tangent of steel is equal to $E_{0}=205,000 \mathrm{MPa}$, and the yield strength is $F_{y}=235 \mathrm{MPa}$, the strain hardening ratio (ratio between post-yield tangent and initial tangent) is 0.01 .20 ground acceleration histories were used among the set of 60 ground motion histories selected by FEMA from the 1994 Northridge earthquake [25]. These accelerations are corresponding to the subset of $10 \%$ exceeding probability for 50 years. They were then scaled with the PGA that was assumed to be log-normal distribution with mean value equal to $2.4 \mathrm{~m} / \mathrm{s}^{2}$ and $\log$ arithmic standard deviation equal to $0.4 \log \left(\mathrm{m} / \mathrm{s}^{2}\right)$. Structural responses were obtained using the OpenSees finite element software [26]. In order to apply the proposed method based on PDEM, the probability density function $p_{A}(a)$ was discretized in 50 points. It is thus necessary to perform $20 \times 50=1000$ mechanical simulations. The accuracy of the proposed method is verified in comparing to the results of the MCS method with $10^{5}$ simulations. The failure of the frame depends on the interstorey drift i.e the relative horizontal displacement of two adjacent floors. Under the 


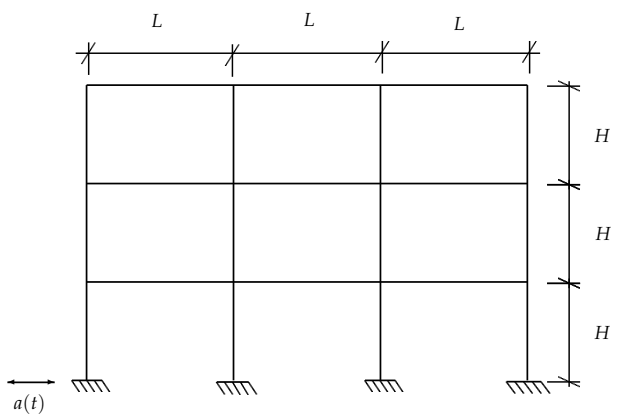

(a)

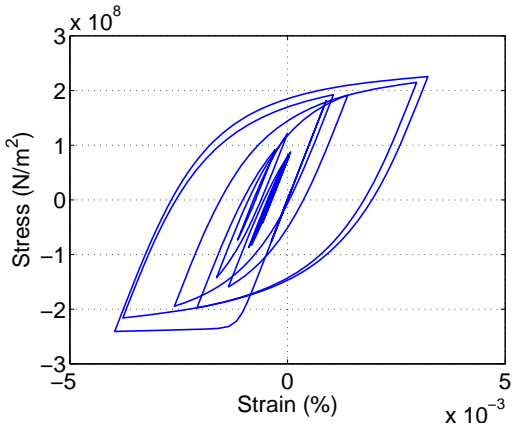

(b)

Fig. 5. Steel frame structure (a) and nonlinear material behavior(b)

excitation of a ground acceleration $a_{g}(t)$, the inter-storey drift of the $k^{\text {th }}$ storey is defined as $\delta^{k}(t)=x^{k}(t)-x^{k-1}(t), k=1,2 \ldots 3$. In comparison with the definition in Eq. (1), the failure happens if $X=\max _{t, k}\left|\delta^{k}(t)\right| \geq x_{0}=\delta_{0}$, where $\delta_{0}$ is the seismic resistance in terms of the inter-storey drift limit of the frame. Three cases were considered: threshold 1: $\delta_{0}=H / 300$, threshold 2: $\delta_{0}=H / 200$ and threshold 3: $\delta_{0}=H / 100$.

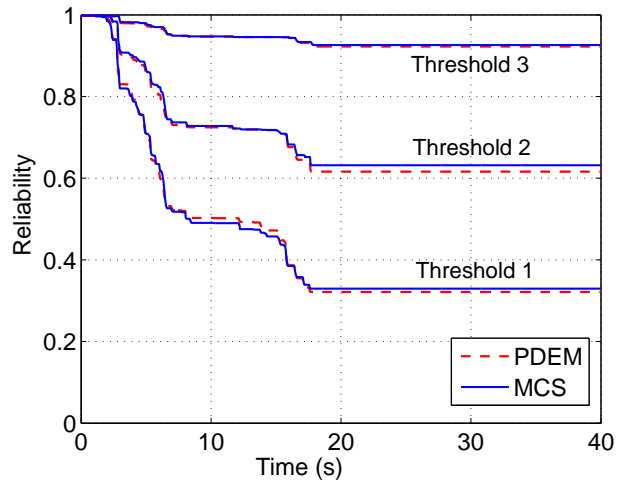

(a)

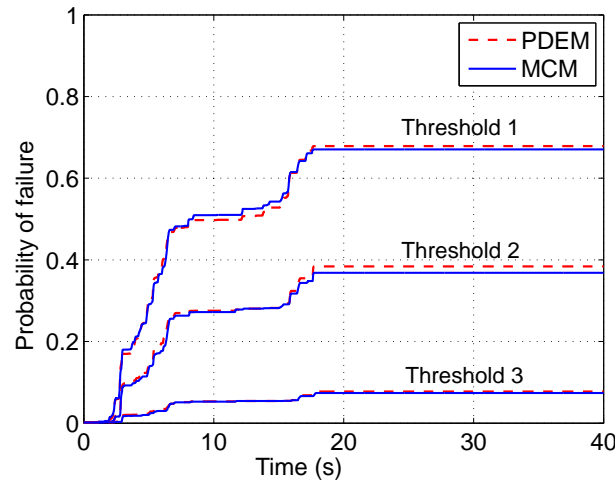

(b)

Fig. 6. Steel frame structure: reliability (a) and probability of failure (b)

Fig. 6 presents the reliability $R(t)$ in the time interval $[0,40 \mathrm{sec}]$ by the PDEM and MCS methods. Similar to the previous example, a good agreement is observed between the PDEM results and those of the MCS.

Fragility surfaces of the frame, corresponding to three critical thresholds $\delta_{0}$ obtained by the proposed method, are presented in Fig. 7. Three fragility curves $F_{r}(a)$ were then derived by using Eq. (17). These curves depicted in Fig. 7, are close to the MCS results. For comparison purpose, the fragility curves derived from the MLE method with 
1000 simulations are also given. They are however less close to the MCS results than those of the proposed method.
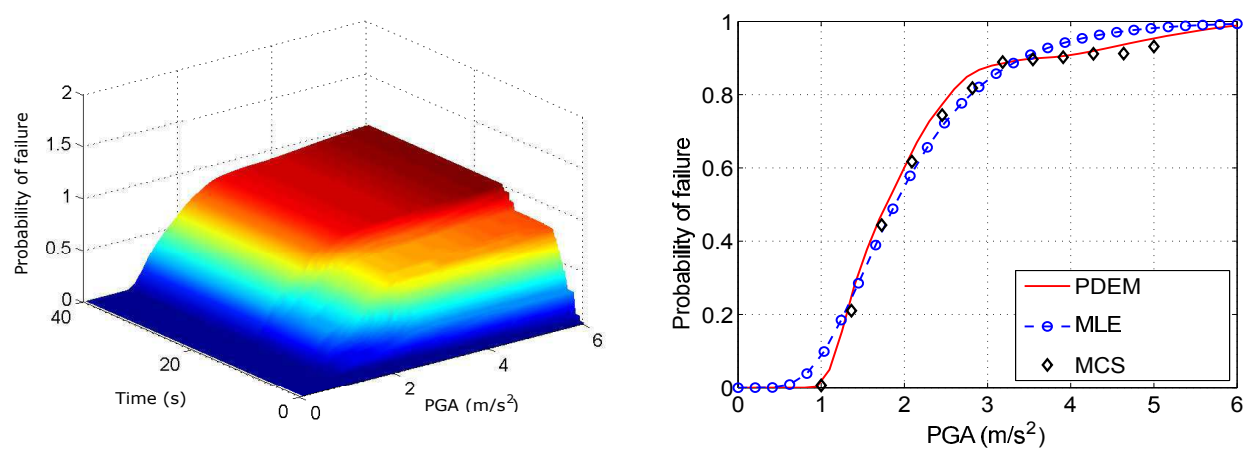

(a) $\delta_{0}=H / 300$
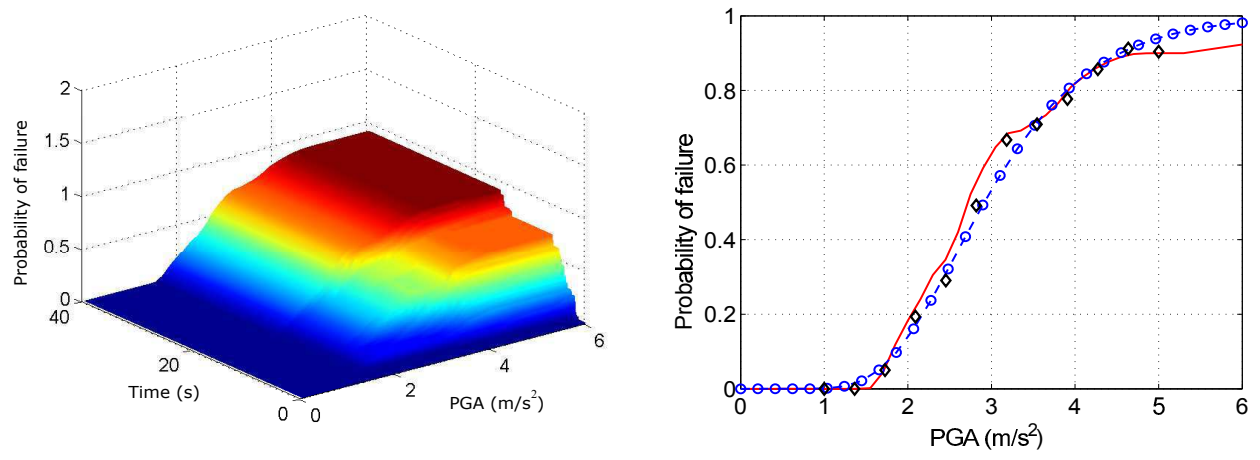

(b) $\delta_{0}=H / 200$
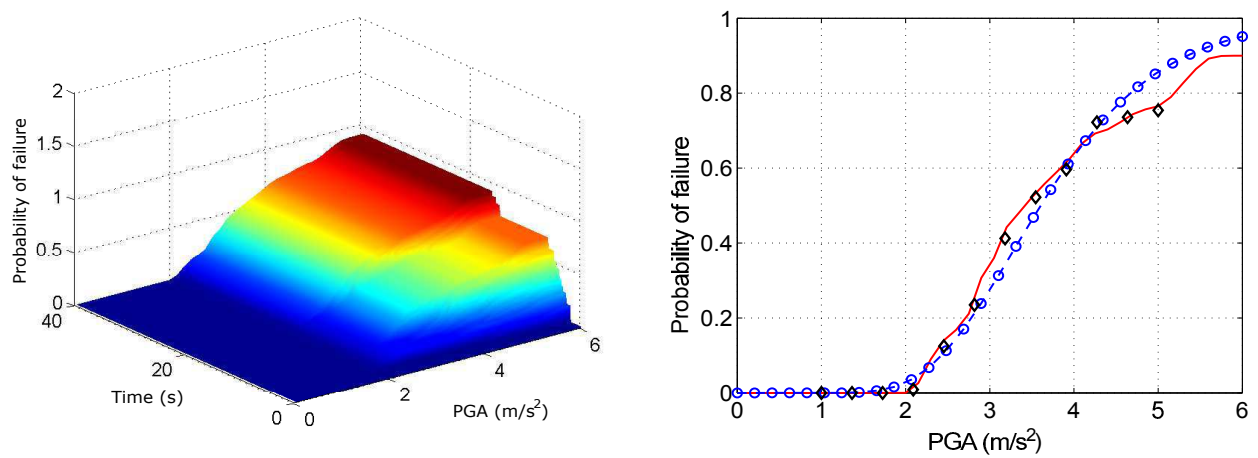

(c) $\delta_{0}=H / 100$

Fig. 7. Steel frame structure: fragility surfaces and fragility curves 


\section{CONCLUSIONS}

The fragility curves are an efficient tool for seismic design, probabilistic seismic risk assessment and quick loss estimation after an earthquake. Based on the assumption of log-normal probability cumulative function for the shape of fragility curves, classical methods aimed to identify two parameters of log-normal distribution from numerical simulation or real observation data. Therefore, they can be denoted as parametric methods.

In this study, a novel non-parametric method for constructing fragility curves, was proposed. Based on the probability density evolution method, the proposed method allows to build fragility curves without the log-normal assumption. It is a novelty of the proposed method compared to existing methods. Moreover, instantaneous fragility curves (or fragility surface) considered as useful complementary information were also available. Based on analytical formulations, a practical step-by-step procedure was proposed.

Two non-linear oscillators and a steel frame were used for validation of the proposed method by comparing its results to those of the MCS method when a high number of simulations was used. In all examples, a very good agreement was noted. It is noteworthy that the proposed method needs much less simulations than the MCS method (600 or 1000 vs. $10^{5}$ ). For comparison purpose, the MLE method was also used with the same number of simulations as the proposed method. Fragility curves derived by the proposed method are closer to the MCS results than those of the MLE method. This shows a good efficiency of the proposed method in the two treated examples. In addition, the fragility surfaces provided by the proposed method can give useful information of safety/failure level of structures during earthquakes.

Finally, it should be noted that in the numerical examples, only uncertainty in earthquake excitations was considered. More random parameters in excitations and in mechanical system properties should be further studied to verify the efficiency of the proposed method.

\section{REFERENCES}

[1] B. Ellingwood. Validation studies of seismic PRAs. Nuclear Engineering and Design, 123, (1990), pp. 189-196. doi:10.1016/0029-5493(90)90237-r.

[2] B. R. Ellingwood. Earthquake risk assessment of building structures. Reliability Engineering E System Safety, 74, (3), (2001), pp. 251-262. doi:10.1016/s0951-8320(01)00105-3.

[3] K. Karim and F. Yamazaki. Effect of earthquake ground motions on fragility curves of highway bridge piers based on numerical simulation. Earthquake Engineering \& Structural Dynamics, 30, (12), (2001), pp. 1839-1856. doi:10.1002/eqe.97.

[4] J. E. Padgett and R. DesRoches. Methodology for the development of analytical fragility curves for retrofitted bridges. Earthquake Engineering \& Structural Dynamics, 37, (8), (2008), pp. 1157-1174. doi:10.1002/eqe.801.

[5] C. Zhou, X. Zeng, Q. Pan, and B. Liu. Seismic fragility assessment of a tall reinforced concrete chimney. The Structural Design of Tall and Special Buildings, 24, (2015), pp. 440-460. doi:10.1002/tal.1173. 
[6] B. S. Ju and A. Gupta. Seismic fragility of threaded Tee-joint connections in piping systems. International Journal of Pressure Vessels and Piping, 132-133, (2015), pp. 106-118. doi:10.1016/j.ijpvp.2015.06.001.

[7] S. A. Argyroudis and K. D. Pitilakis. Seismic fragility curves of shallow tunnels in alluvial deposits. Soil Dynamics and Earthquake Engineering, 35, (2012), pp. 1-12. doi:10.1016/j.soildyn.2011.11.004.

[8] S. Argyroudis and A. M. Kaynia. Analytical seismic fragility functions for highway and railway embankments and cuts. Earthquake Engineering $\mathcal{E}$ Structural Dynamics, 44, (2015), pp. 1863-1879. doi:10.1002/eqe.2563.

[9] K. Karim and F. Yamazaki. A simplified method of constructing fragility curves for highway bridges. Earthquake Engineering \& Structural Dynamics, 32, (10), (2003), pp. 1603-1626. doi:10.1002/eqe.291.

[10] C. Kafali and M. Grigoriu. Seismic fragility analysis: Application to simple linear and nonlinear systems. Earthquake Engineering \& Structural Dynamics, 36, (13), (2007), pp. 1885-1900. doi:10.1002/eqe.726.

[11] M. Shinozuka, M. Feng, H. Kim, and S. Kim. Nonlinear static procedure for fragility curve development. Journal of Engineering Mechanics, 126, (12), (2000), pp. 1287-1295. doi:10.1061/(asce)0733-9399(2000)126:12(1287).

[12] M. Shinozuka, M. Feng, J. Lee, and T. Naganuma. Statistical analysis of fragility curves. Journal of Engineering Mechanics, 126, (12), (2000), pp. 1224-1231. doi:10.1061/(asce)07339399(2000)126:12(1224).

[13] M. Shinozuka, S. Kim, S. Kushiyama, and J. Yi. Fragility curves of concrete bridges retrofitted by column jacketing. Earthquake Engineering and Engineering Vibration, 1, (2), (2002), pp. 195205. doi:10.1007/s11803-002-0065-2.

[14] A. Cornell, F. Jalayer, R. Hamburger, and D. Foutch. Probabilistic basis for 2000 sac federal emergency management agency steel moment frame guidelines. Journal of Structural Engineering, 128, (4), (2002), pp. 526-533. doi:10.1061/(asce)0733-9445(2002)128:4(526).

[15] T.-P. Le, C.-T. Dang, and P. Ray. A comparative study of construction methods for seismic fragility curves using numerical simulations. Mechanics and Industry, 17, (6), (2016), p. 12. doi:http:/ /dx.doi.org/10.1051/meca/2015119.

[16] J. Li and J. B. Chen. Probability density evolution method for dynamic response analysis of structures with uncertain parameters. Computational Mechanics, 34, (5), (2004), pp. 400-409. doi:10.1007/s00466-004-0583-8.

[17] J. B. Chen and J. Li. Dynamic response and reliability analysis of non-linear stochastic structures. Probabilistic Engineering Mechanics, 20, (1), (2005), pp. 33-44. doi:10.1016/j.probengmech.2004.05.006.

[18] J. Li and J. B. Chen. The probability density evolution method for dynamic response analysis of non-linear stochastic structures. International Journal for Numerical Methods in Engineering, 65, (6), (2006), pp. 882-903. doi:10.1002/nme.1479.

[19] J. B. Chen and J. Li. Joint probability density function of the stochastic responses of nonlinear structures. Earthquake Engineering and Engineering Vibration, 6, (2007), pp. 35-47. doi:10.1007/s11803-007-0672-z.

[20] J. Li and J. B. Chen. The principle of preservation of probability and the generalized density evolution equation. Structural Safety, 30, (1), (2008), pp. 65-77. doi:10.1016/j.strusafe.2006.08.001.

[21] D. M. Boore. Simulation of ground motion using the stochastic method. Pure and Applied Geophysics, 160, (3), (2003), pp. 635-676. doi:10.1007/978-3-0348-8010-7_10. 
[22] G. M. Atkinson and W. Silva. Stochastic modeling of California ground motions. Bulletin of the Seismological Society of America, 90, (2), (2000), pp. 255-274. doi:10.1785/0119990064.

[23] MATLAB. version 7.9.0529 (R2009b). The MathWorks Inc., Natick, Massachusetts, (2009).

[24] B. Sudret and C. Mai. Calcul des courbes de fragilité sismique par approches nonparamétriques. In Proceedings of Congrès Francais de Mécanique 2013, Bordeaux, France, (2013), https: / / www.research-collection.ethz.ch/bitstream/handle/20.500.11850/154294/eth7983-01.pdf?sequence=1\&isAllowed=y.

[25] J.-H. Yi, S.-H. Kim, and S. Kushiyama. PDF interpolation technique for seismic fragility analysis of bridges. Engineering Structures, 29, (7), (2007), pp. 1312-1322. doi:10.1016/j.engstruct.2006.08.019.

[26] S. Mazzoni, F. McKenna, M. H. Scott, G. L. Fenves, and B. Jeremic. Open system for earthquake engineering simulation (OpenSees). User Command Language Manual, Version, 1, (3), (2006). 